\title{
ON THE COVERING RADIUS PROBLEM FOR CODES I. BOUNDS ON NORMALIZED COVERING RADIUS*
}

\author{
KAREN E. KILBY† AND N. J. A. SLOANE $\ddagger$
}

\begin{abstract}
In this two-part paper we introduce the notion of a stable code and give a new upper bound on the normalized covering radius of a code. The main results are that, for fixed $k$ and large $n$, the minimal covering radius $t[n, k]$ is realized by a normal code in which all but one of the columns have multiplicity 1 ; hence $t[n+2, k]=t[n, k]+1$ for sufficiently large $n$. We also show that codes with $n \leqq 14, k \leqq 5$ or $d_{\min } \leqq 5$ are normal, and we determine the covering radius of all proper codes of dimension $k \leqq 5$. Examples of abnormal nonlinear codes are given. In Part I we investigate the general theory of normalized covering radius, while in Part II [this Journal, 8 (1987), pp. 619-627] we study codes of dimension $k \leqq 5$, and normal and abnormal codes.
\end{abstract}

Key words. binary codes, covering radius

AMS(MOS) subject classifications. 05B, 94B

1. Introduction. Let $C$ be an $[n, k]$ binary linear code. The covering radius $R$ (also denoted by $C R(C)$ ) is given by

$$
R=\max _{x \in \mathbf{F}^{n}} \min _{c \in C} d(x, c),
$$

where $\mathbf{F}=\{0,1\}$ and $d(, \quad)$ is Hamming distance. Let $t[n, k]$ denote the smallest $R$ for any $[n, k]$ code. Two central problems in this subject are to determine $t[n, k]$ and to construct codes with $R=t[n, k]$ (see [1]-[3], [9], [10] for further background information).

Before describing the new results, we define the normalized covering radius, which as we shall see is easier to work with than the covering radius itself. Let $C$ have generator matrix $G$. In general, $G$ may contain repeated columns. We assume throughout, however, that no column of $G$ is zero. Let $a$ be the number of distinct columns occurring in $G$, and let $m_{1}, \cdots, m_{a}$ be their multiplicities, with $m_{1}+\cdots+m_{a}=n$. Then

$$
R \geqq \sum_{i=1}^{a}\left[\frac{m_{i}}{2}\right] \text {, }
$$

and, following [10], we define the normalized covering radius $\rho$ of $C$ to be

$$
\rho=R-\sum_{i=1}^{a}\left[\frac{m_{i}}{2}\right]
$$

a nonnegative integer. Then

$$
R=\sum_{i=1}^{a}\left[\frac{m_{i}}{2}\right]+\rho=\frac{n}{2}-\frac{\text { no. of odd } m_{i}}{2}+\rho .
$$

Summary of results. A stable code $(\S 3)$ has the property that $\rho$ does not increase when any number of pairs of identical columns of any length are adjoined to it. Many small codes are stable ( $\$ 6$ of Part II), so this often provides a quick method for determining the covering radius. The contracted code $\tilde{C}(\S 3)$ is spanned by the rows of the matrix formed by taking one copy of each column of $G$ that has odd multiplicity, where $G$ is

* Received by the editors February 6, 1986; accepted for publication (in revised form) March 24, 1987.

$\dagger$ Morse College, Yale University, New Haven, Connecticut 06520. The work of this author was supported in part by Bell Laboratories.

‡ Mathematical Sciences Research Center, AT\&T Bell Laboratories, Murray Hill, New Jersey 07974. 
a generator matrix for $C$. If $\tilde{C}$ is stable, $\rho(C)=\rho(\tilde{C})$. As an illustration, consider the code $C$ with generator matrix

\begin{tabular}{|c|c|c|c|}
\hline 111 & 000 & 00 & 00 \\
\hline 00 & 111 & 00 & 00 \\
\hline 00 & 000 & 11 & 00 \\
\hline 000 & 000 & 00 & 11 \\
\hline
\end{tabular}

(with multiplicities 3, 3, 2, 2, 2), encountered in the proof of Theorem 27 of [2]. The contracted code $\tilde{C}$ has generator matrix $\left(\begin{array}{ll}1 & 0 \\ 0 & 1\end{array}\right)$, and is stable with $\rho=0$, so $C$ has covering radius $0+\sum\left[m_{i} / 2\right]=5$. Stable codes are normal (see Theorem 4 ).

Theorems 6 and 7 give improved upper bounds on $\rho$. Section 5 considers how $\rho$ increases when the multiplicities of the columns are increased, subject to the constraint that the parities of the multiplicities are unchanged. More precisely, fix an $\left[n_{B}, k\right]$ projective code $B$ (i.e., one with distinct columns), and consider all $[n, k] \operatorname{codes} C$ with $\tilde{C}=B$. For sufficiently large $n, \rho_{\infty}(B)=\max _{C} \rho(C)$ and $\rho_{*}(B)=\min _{C} \rho(C)$ are independent of $n$. Theorem 8 investigates how rapidly $\rho_{\infty}(B)$ can be reached. Theorem 9 shows that $\rho_{*}(B)$ can be realized by a normal code having a very special structure, in which all columns have multiplicity 1 except for one column that has large multiplicity. Furthermore a normal code $C$ has $\rho_{*}(C)=\rho(C)$ (see Theorem 11).

For fixed $k$ and large $n$, the minimal covering radius of any $[n, k]$ code is given by

$$
t[n, k]=\frac{n}{2}+\min _{B}\left\{\rho_{*}(B)-\frac{n_{B}}{2}\right\},
$$

where $B$ ranges over the projective codes of dimension $k$ or $k-1$ (equation (52)). It follows (see Theorem 12) that, for large $n, t[n, k]$ can be attained by a normal code having the above-mentioned special structure. This establishes Conjectures A and D of [2] for sufficiently large $n$. A heuristic justification for the special structure of these codes is given at the end of $\S 6$ of Part II.

Codes of dimension $k \leqq 4$ were studied in [10]. We have now determined the covering radius of every projective code of dimension 5 . If $C$ is any $[n, 5]$ code, then

$$
C R(\tilde{C}) \leqq \rho(C) \leqq C R(\tilde{C})+1
$$

(see Theorem 13 of Part II). This implies that all codes of dimension $\leqq 5$ are normal.

Table 2(a) of Part II gives upper and lower bounds on $\rho$, and enables one to write down the covering radius of any code $C$ for which the contracted code $\tilde{C}$ has dimension $\leqq 5$, with an error of at most 1 , when only the length and dimension of $\tilde{C}$ are known. For example, suppose $C$ is a $[3000,12]$ code for which $\tilde{C}$ is a $[20,5]$ code. From Table 2(a) of Part II we see that $7 \leqq \rho(C) \leqq 9$, or in other words (using (2))

$$
C R(C)=\frac{3000}{2}-\frac{20}{2}+8+\theta=1498+\theta,
$$

where $\theta=-1,0$ or 1 .

Section 6 presents a summary of the projective codes of dimension 5 and length $5 \leqq n \leqq 31$, and gives one or two examples of the best covering codes of each length (see especially Fig. 3 of Part II). This list of codes should be useful, since the investigation of the subject has been hampered by a shortage of examples of good covering codes. The precise determination of $\rho$ for some of these codes requires a separate analysis, as illustrated in Theorem 10, and we have only carried this out in certain cases. At the end of $\S 6$ of Part II we construct an infinite family of (normal) codes with unacceptable coordinates. 
Sections 7 and 8 of Part II show that if $d_{\min } \leqq 5$ or if $\tilde{C}$ has $d_{\min } \leqq 2$ then $C$ is normal. Theorem 18 of Part II summarizes the known conditions on the parameters of a code that imply normality. Finally, $\S 9$ of Part II gives Peter Frankl's construction of an abnormal nonlinear code.

Definitions and notation. The minimal distance of a code is denoted by $d_{\min }$, and the order of its automorphism group by $g$. We use $\mathbf{F}$ to denote both the Galois field $G F(2)$ and the code $\{0,1\}$. The empty code of length zero will be denoted by 0 , and $E_{n}$ denotes the $[n, n-1] R=1$ even weight code. The $[n, 1] R=\left[\frac{1}{2} n\right]$ repetition code $T_{n}$ contains $0^{n}$ and $1^{n}$. The $\left[n=2^{k}-1, k\right] R=2^{k-1}$ simplex code $S_{k}$ is defined by a generator matrix in which the columns comprise all distinct nonzero binary $k$-tuples (see (14) and Fig. 3 of Part II). In particular, $S_{0}=0, S_{1}=\mathbf{F}, S_{2}=E_{3}$. The $2^{k}-1$ columns of a generator matrix for $S_{k}$, for $k \geqq 3$, may be regarded as representing the points of a projective geometry $P G(2, k-1)$ of dimension $k-1$ over $\mathbf{F}$. In such a geometry every line contains exactly three points; three points are collinear if and only if the corresponding vectors sum to zero. We shall occasionally use this geometrical language even when $k$ is less than 3 .

Normal codes. Let $C$ be a linear or nonlinear code of length $n$ and covering radius $R$. For $i=1, \cdots, n$ and $a=0,1$ let $C_{a}^{(i)}$ denote the subset of codewords $\left(c_{1}, \cdots, c_{n}\right)$ of $C$ with $c_{i}=a$, and for an arbitrary $x \in \mathbf{F}^{n}$ let

$$
f_{a}^{(i)}(x)=d\left(x, C_{a}^{(i)}\right),
$$

if $C_{a}^{(i)}$ is nonempty, and let $f_{a}^{(i)}(x)=n$ otherwise. Then

$$
N^{(i)}=\max _{x}\left\{f_{0}^{(i)}(x)+f_{1}^{(i)}(x)\right\}
$$

is called the norm of $C$ with respect to the ith coordinate. If

$$
N^{(i)} \leqq N
$$

for at least one coordinate $i$, we say that $C$ has norm ${ }^{1} N$, and coordinates $i$ for which (3) holds are called acceptable, the other coordinates being unacceptable. Finally, $C$ is normal if it has norm $N$ satisfying

$$
N \leqq 2 R+1 \text {, }
$$

and is otherwise abnormal. It follows from the definition that if $C$ has norm $N$, it also has norm $N+1, N+2, \cdots$. We take $N$ as small as possible. For any code,

$$
2 R \leqq N \text {. }
$$

Many other properties of the norm will be found in [3].

2. Normalized covering radius $\rho$. Let $C$ be an $[n, k] R$ code (assumed throughout to have no coordinate position that is identically zero). Then [1, Thm. 6]

$$
R \leqq\left[\frac{n}{2}\right] .
$$

The normalized covering radius $\rho(C)$, defined in (1), satisfies (see [10]) $\rho(C) \geqq 0$; if all $m_{i}$ are even, $\rho(C)=0$; if all $m_{i}$ are $1, \rho(C)=R$. (Note that $\rho(C)$ does not depend on the

${ }^{1}$ The meanings of three terms in this paper differ from those used in certain earlier works, as will be indicated in footnotes. The new definitions seem preferable. This definition of norm is the one used in [2], [10] and the manuscript of [3]. The definition given in the published version of [3] turns out to be less satisfactory. 
choice of a generator matrix.) By reordering the coordinates (if necessary) we may assume that the first $m_{1}$ columns of $C$ are identical, then the next $m_{2}$ columns, and so on. We partition the codewords $c \in C$ as

$$
c=\left(c^{(1)}, c^{(2)}, \cdots, c^{(a)}\right), \quad c^{(i)}=\left(c_{i}, c_{i}, \cdots, c_{i}\right)
$$

where length $\left(c^{(i)}\right)=m_{i}$. Correspondingly, we partition an arbitrary vector $x \in \mathbf{F}_{2}^{n}$ as

$$
x=\left(x^{(1)}, \cdots, x^{(a)}\right),
$$

where length $\left(x^{(i)}\right)=m_{i}$. The height of $x^{(i)}$ is defined to be

$$
h_{i}=h t\left(x^{(i)}\right)=w t\left(x^{(i)}\right)-\left[\frac{m_{i}}{2}\right] \text {, }
$$

and the height of $x$ is

$$
h t(x)=\sum_{i=1}^{a} h_{i}
$$

Then we have $([10,(15)])$

$$
\rho(C)=\max _{x} \min _{c \in C} h t(x+c) .
$$

A vector $x$ such that $\min _{c \in C} h t(x+c)=\rho(C)$, or equivalently $d(x, C)=R$, is called a deep hole in $C$. It is shown in Theorem 1 of [10] that, using (11), we can express the problem of finding $\rho(C)$ as an integer programming problem.

The following result will turn out to be very useful.

THEOREM 1. Suppose a code $C$ is the row space of a matrix of the form $\left[\begin{array}{ll}G_{1} & G_{3} \\ 0 & G_{2}\end{array}\right]$, where $G_{1}$ and $G_{2}$ have no columns of 0 's, and let codes $A$ and $B$ be the row spaces of $G_{1}$ and $G_{2}$, respectively. (a) If $G_{3}=0$, so that $C$ is a direct sum $C=A \oplus B$, then

$$
\rho(C)=\rho(A)+\rho(B),
$$

and if either $A$ or $B$ is normal so is $C$. (b) If all columns in $\left[G_{G_{2}}^{G_{3}}\right]$ occur an even number of times, then $\rho(C)=\rho(A)$, and if $A$ is normal so is $C$.

Proof. (a) Clearly

$$
C R(C) \leqq C R(A)+C R(B)
$$

(cf. [7]), with equality if $G_{3}=0$, which implies (12). Suppose $G_{3}=0, A$ is normal and coordinate $r$ is acceptable. Let $A, B, C$ have lengths $n_{A}, n_{B}, n_{C}$, respectively. For an arbitrary $x=|y| z \mid \in \mathbf{F}^{n_{C}}, y \in \mathbf{F}^{n_{A}}, z \in \mathbf{F}^{n_{B}}$ we have, in the notation of $\S 1$,

$$
\begin{aligned}
d\left(x, C_{a}^{(r)}\right) & =d\left(y, A_{a}^{(r)}\right)+d(z, B), \quad a=0,1, \\
\operatorname{Norm}(C) & =\operatorname{Norm}(A)+2 C R(B) \\
& \leqq 2 C R(A)+1+2 C R(B) \\
& =2 C R(C)+1,
\end{aligned}
$$

and $C$ is normal. (b) Now $C R(C)=C R(A)+\frac{1}{2} n_{B}$, so $\rho(C)=\rho(A)$. If $A$ is normal, then $d\left(x, C_{a}^{(r)}\right) \leqq d\left(y, A_{a}^{(r)}\right)+\frac{1}{2} n_{B}$, and again Norm $(C) \leqq 2 C R(C)+1$.

3. The effect on $\rho$ of varying the multiplicities; stable codes. We now investigate how $\rho(C)$ changes as the multiplicities $m_{i}$ of the columns of $C$ vary. Any code $C$ of dimension $\leqq k$ can be obtained by assigning suitable multiplicities to the columns of the simplex code $S_{k}$. Let us arrange the columns of $S_{k}$ in some fixed order, for example, the 
binary order illustrated in (14) for $S_{3}$ (see also Fig. 3 of Part II):

$$
S_{3}:\left[\begin{array}{lllllll}
0 & 0 & 0 & 1 & 1 & 1 & 1 \\
0 & 1 & 1 & 0 & 0 & 1 & 1 \\
1 & 0 & 1 & 0 & 1 & 0 & 1
\end{array}\right] \text {. }
$$

We choose a generator matrix $G$ for $C$, and let $m_{i} \geqq 0$ be the number of times the $i$ th column of $S_{k}$ appears in $G$, for $i=1, \cdots, 2^{k}-1$. Then we let

$$
\rho^{(k)}\left(m_{1}, m_{2}, \cdots, m_{2^{k}-1}\right)
$$

equal the normalized covering radius $\rho(C)$. Choosing a different generator matrix for $C$ permutes the $m_{i}$ 's but does not change the value of $\rho^{(k)}\left(m_{1}, \cdots, m_{2^{k}-1}\right)$. (However most permutations of the arguments do change the value of $\rho^{(k)}\left(m_{1}, \cdots, m_{2^{k}-1}\right)$.)

It turns out that the function $\rho^{(k)}$ is best studied by allowing the $m_{i}$ to vary while fixing their parity, or in other words by investigating how $\rho^{(k)}$ changes when pairs of identical columns are added to or deleted from the generator matrix $G$. It is an elementary fact that when two identical columns are adjoined to $G$ (columns which may or may not already be present in $G$ ), the covering radius of $C$ increases by either 1 or 2 , so the normalized covering radius is either unchanged or increases by 1 . This establishes the monotonicity property [10, Thm. 2]: if $m_{i} \leqq m_{i}^{\prime}$ and $m_{i} \equiv m_{i}^{\prime}(\bmod 2)$ for all $i$, then

$$
\rho^{(k)}\left(m_{1}, \cdots, m_{2^{k}-1}\right) \leqq \rho^{(k)}\left(m_{1}^{\prime}, \cdots, m_{2^{k}-1}^{\prime}\right) .
$$

The earliest codes to be considered are therefore the projective codes. Given an arbitrary code $C$, with parameters $[n, k] R$ and generator matrix $G$, the corresponding contracted code ${ }^{2} \tilde{C}$ is the projective code which is the row space of the matrix formed by taking one copy of each column of $G$ that has odd multiplicity. $\tilde{C}$ is independent of the choice of $G$. (If all $m_{i}$ are even we set $\tilde{C}$ equal to the empty code 0 .) We denote the parameters of $\tilde{C}$ by $[\tilde{n}, \tilde{k}] \tilde{R}$, so $\tilde{n} \leqq n, \tilde{k} \leqq k, \tilde{R} \leqq R$ and $\rho(\tilde{C}) \leqq \rho(C)$.

We say that two codes $C, D$ are congruent (written $C \equiv D$ ) if $\tilde{C}=\tilde{D}$. If the multiplicities of the columns in $C$ do not exceed the multiplicities of the same columns in $D$ (but with no constraints on their parities), we write $C \leqq D$. For example, the contracted code $\tilde{C}$ satisfies

$$
\tilde{C} \equiv C \quad \text { and } \quad \tilde{C} \leqq C .
$$

The monotonicity property (15) states that

$$
C \equiv D, \quad C \leqq D \quad \Rightarrow \quad \rho(C) \leqq \rho(D) .
$$

We shall need the following corollary to Theorem 19 of [3].

THEOREM 2. Let $C$ be normal and suppose the rth coordinate is acceptable. Let $D$ be formed by adjoining $2 m$ copies of the rth coordinate to $C$. Then $C R(D)=C R(C)+$ $m$, Norm $(D)=$ Norm $(C)+2 m, D$ is normal, and any copy of the rth coordinate is an acceptable coordinate for $D$.

Proof. $D$ is an amalgamated direct sum [3] of $C$ with the repetition code $T_{2 m+1}$. By the remarks made earlier in this section, $C R(D) \geqq C R(C)+m$. From Theorem 19(ii) of [3], $C R(D)=C R(C)+m$. Then $D$ is normal by Theorem 19(iii) of [3].

The determination of the covering radius of codes of low dimension is greatly facilitated by the observation that for many of these codes $\rho$ does not increase when pairs of identical columns are adjoined to the generator matrix. We call $C$ stable if it has this

\footnotetext{
${ }^{2} \mathrm{~A}$ different definition of contracted code was used in [2].
} 
property, or more precisely if

$$
C \equiv D, \quad C \leqq D \quad \Rightarrow \quad \rho(C)=\rho(D) .
$$

We shall see, for example, that all codes of dimension $k \leqq 3$ are stable.

In view of Theorem 1(b), adjoining pairs of identical columns from outside the subspace of $P G(2, k-1)$ spanned by the columns of $C$ has no effect on $\rho$, so such columns can be ignored when investigating the stability of $C$.

THEOREM 3. Let an $[n, k]$ code $C$ be the row space of a matrix $G$, and let $\Pi$ be the subspace of $P G(2, k-1)$ spanned by the columns of $G$. Then $C$ is stable if and only if $\rho(C)$ does not increase when any number of pairs of identical columns representing points in $\Pi$ are adjoined to $G$.

Examples. If all the multiplicities $m_{i}$ are even, then $\rho=0$. But $\tilde{C}$ is the empty code 0 , with $\rho=0$. We deduce that 0 is stable. (This can also be deduced directly from the theorem.)

If $C$ has dimension 1 then (since no coordinate may be identically zero) $C=T_{n}$, $R=[n / 2], \rho=0$. But $\tilde{T}_{n}=0$ if $n$ is even, $\tilde{T}_{n}=\mathbf{F}$ if $n$ is odd, both having $\rho=0$. We deduce that $\mathbf{F}$ is stable. In fact all the codes $\mathbf{F}^{n}$ and $E_{n}$ are stable (see the examples preceding Theorem 6).

Any code $C$ of dimension 2 has a generator matrix containing (say) $a$ columns $\left(\begin{array}{l}0 \\ 1\end{array}\right)$, $b$ columns $\left(\begin{array}{l}1 \\ 0\end{array}\right)$ and $c$ columns $\left(\begin{array}{l}1 \\ 1\end{array}\right)$. As stated on page 388 of [3], the covering radius of $C$ is given by

$$
\begin{aligned}
& {\left[\frac{a}{2}\right]+\left[\frac{b}{2}\right]+\left[\frac{c}{2}\right]+1 \quad \text { if } a, b, c \text { are odd, }} \\
& {\left[\frac{a}{2}\right]+\left[\frac{b}{2}\right]+\left[\frac{c}{2}\right] \quad \text { otherwise. }}
\end{aligned}
$$

This now has a very short proof. We calculate $\tilde{C}$, which is

$$
\begin{aligned}
& 0, \text { with } \rho=0, \quad \text { if } a, b, c \text { are even, } \\
& \mathbf{F} \text {, with } \rho=0, \quad \text { if one of } a, b, c \text { is odd, } \\
& \mathbf{F}^{2} \text {, with } \rho=0, \quad \text { if two of } a, b, c \text { are odd, } \\
& E_{3} \text {, with } \rho=1, \quad \text { if } a, b, c \text { are odd. }
\end{aligned}
$$

All four codes are stable, and (19), (20) follow immediately.

THEOREM 4. A stable code is normal, and all coordinates are acceptable.

LEMMA 5. Let $C$ be any $[n, k]$ code such that for some $i(1 \leqq i \leqq n)$, and all $l=$ $0,1,2, \cdots$, adjoining $2 l$ copies of column i to $C$ does not increase the normalized covering radius $\rho$. Then $C$ is normal and coordinate $i$ is acceptable.

Proof. Suppose coordinate $i$ is unacceptable. Therefore there is a vector $x$ such that $f_{0}^{(i)}(x)+f_{1}^{(i)}(x) \geqq 2 R+2$. Without loss of generality $f_{0}^{(i)}(x) \leqq f_{1}^{(i)}(x)$, say $f_{0}^{(i)}(x)=R-\theta$, $f_{1}^{(i)}(x) \geqq R+\theta+2$, where $0 \leqq \theta \leqq R$. We construct $D$ by adjoining $2 R+2$ copies of column $i$ to $C$. Then $\rho(D)=\rho(C)$, so

$$
C R(D)=C R(C)+R+1=2 R+1 .
$$

Let $x^{*}=|x| u \mid$, where $u$ is a vector of length $2 R+2$ and weight $w=R+\theta+2$. Then for $D$,

$$
\begin{aligned}
& f_{0}^{(i)}\left(x^{*}\right)=f_{0}^{(i)}(x)+w=2 R+2, \\
& f_{1}^{(i)}\left(x^{*}\right)=f_{1}^{(i)}(x)+2 R+2-w \geqq 2 R+2,
\end{aligned}
$$

which contradicts $(21)$. 
Theorem 4 now follows because a stable code satisfies the hypothesis of the lemma for every $i$.

4. Upper bounds on $\rho$. Suppose $C$ is an $[n, k] R$ code, and the contracted code $\tilde{C}$ is an $[\tilde{n}, \tilde{k}] \tilde{R}$ code. It follows immediately from (1), (6) that

$$
\rho(C) \leqq\left[\frac{\tilde{n}}{2}\right] .
$$

A stronger upper bound was given in Theorem 8 of [10]. The main goal of the present section is to prove Theorem 7, which strengthens both of these results. We first quote (from Corollary 6 of [7]) the result that the maximal covering radius of any $[n, k]$ code is given by

$$
T[n, k]= \begin{cases}{\left[\frac{n}{2}\right]} & \text { for } \quad 1 \leqq k \leqq\left[\frac{n}{2}\right], \\ n-k & \text { for }\left\lceil\frac{n}{2}\right\rceil \leqq k \leqq n\end{cases}
$$

where $\lceil x\rceil$ denotes the smallest integer $\geqq x$.

The method of pivoting, introduced in $\S$ VII of [10], is a useful technique for getting upper bounds on $\rho(C)$ (which are often tight), and leads to Theorem 6. Consider $C$ to be formed from the simplex code $S_{k}$ with appropriate multiplicities $m_{i}$, with length $n=$ $\sum m_{i}$. We partition vectors of $\mathbf{F}^{n}$ into blocks as in (7), (8). We choose a coordinate $Q\left(1 \leqq Q \leqq 2^{k}-1\right)$, called the pivot, such that $m_{Q} \neq 0$. For an arbitrary vector $x$ we first make $h t\left(x^{(Q)}\right) \leqq 0$ by (if necessary) adding a codeword $c=\left(c^{(1)}, \cdots, c^{\left(2^{k}-1\right)}\right) \in C$ for which $c^{(Q)} \neq 0$.

Let $C_{a}^{[Q]}$ denote the set of all codewords of $C$ for which $c^{(Q)}=a$, with the $Q$ th block of coordinates deleted, for $a=0,1 . C_{0}^{[Q]}$ is a code of length $n-m_{Q}$ and dimension $k-1 . C_{1}^{[Q]}$ is a translate of $C_{0}^{[Q]}$ and has the same covering radius. In particular, $C_{0}^{[Q]}$ is obtained by assigning multiplicities $m_{P}^{\prime}$ (say) to $S_{k-1}$. The $m_{P}^{\prime}$ are related to the original multiplicities $m_{P}$ as follows. The $m_{P}\left(1 \leqq P \leqq 2^{k}-1\right)$ are nonnegative integers assigned to the points $P \in P G(2, k-1)$. When we form the subcode $C_{0}^{[Q]}$, the $m_{P}$ are combined in pairs to give the new multiplicities $m_{P}^{\prime}$. The multiplicities $m_{R}$ and $m_{S}$ are combined if and only if $Q R S$ is a line in $P G(2, k-1)$. Thus

$$
m_{P}^{\prime}=m_{R}+m_{S} \text { for } Q R S \text { a line in } P G(2, k-1) .
$$

In particular, the number of distinct columns in $C_{0}^{[Q]}$ with odd multiplicity, $\nu$ say, is equal to the number of lines $Q T U$ for which one of $m_{T}$ and $m_{U}$ is odd and the other even.

We return to the problem of reducing the distance from $x$ to $C$. By adding a suitable codeword of $C_{0}^{[Q]}$ we can make $h t(x) \leqq \rho^{(k-1)}\left(m_{1}^{\prime}, \cdots, m_{2^{k-1}-1}^{\prime}\right)$. This leads to the pivoting bound [10, Thm. 7]: if $m_{Q} \neq 0$,

$$
\rho(C) \leqq \eta+\rho\left(C_{0}^{[Q]}\right),
$$

i.e.,

$$
\rho^{(k)}\left(m_{1}, \cdots, m_{2^{k}-1}\right) \leqq \eta+\rho^{(k-1)}\left(m_{1}^{\prime}, \cdots, m_{2^{k-1}-1}^{\prime}\right),
$$

where $\eta$ is the number of lines $Q R S$ for which $m_{R}$ and $m_{S}$ are odd, and the $m_{P}^{\prime}$ are given 
by (25). In the other direction, we have (from (17))

$$
\rho(\tilde{C}) \leqq \rho(C) .
$$

It is often convenient to be able to refer directly to the code obtained by contracting $C_{0}^{[Q]}$, which we shall denote by $\hat{C}$. In particular, if $\hat{C}$ is stable, we have

$$
\rho(C) \leqq \eta+\rho(\hat{C}) .
$$

Remarks. (1) Different choices for the pivot $Q$ may give different bounds, so we can replace the right side of (27) by

$$
\min _{Q}\left\{\eta+\rho^{(k-1)}\left(m_{1}^{\prime}, \cdots, m_{2^{k-1}-1}^{\prime}\right)\right\} .
$$

It appears best to choose $Q$ so that $m_{Q}$ is odd. Even so, (30) may not be tight: there may be no $Q$ for which equality holds in (27). If there is such a $Q$ we call $C$ and $\tilde{C}$ tame, otherwise wild. (2) It was shown in [10] (see especially Theorem 9) that if $\operatorname{dim} C=$ $\operatorname{dim} \tilde{C}$ and the bounds (26) and (28) differ by at most 1, i.e., if, for some choice of the pivot $Q$, either $\rho(\tilde{C})=\eta+\rho\left(C_{0}^{[Q]}\right)$, or $\rho(\tilde{C})=\eta+\rho\left(C_{0}^{[Q]}\right)-1$, then $C$ is normal. (It is easy to show in fact that the hypothesis $\operatorname{dim} C=\operatorname{dim} \tilde{C}$ is unnecessary.)

Examples. Suppose $C$ is an $[n, k]$ code such that $\tilde{C}=\mathbf{F}^{k}$. We show by induction on $k$ that $\rho(C)=0$. Without loss of generality $C$ has a generator matrix $\left[I G_{1}\right]$ where $I$ is an identity matrix and all the columns of $G_{1}$ have even multiplicity. By pivoting on the first coordinate, we obtain $\rho(C)=0$. Therefore $\mathbf{F}^{k}$ is stable for all $k$, and $\rho\left(F^{k}\right)=0$. A similar argument shows that $E_{k}$ is stable and $\rho\left(E_{2}\right)=0, \rho\left(E_{k}\right)=1$ for $k \geqq 3$.

THEOREM 6. Let $C$ be an $[n, k]$ code and let the contracted code $\tilde{C}$ be an $[\tilde{n}, \tilde{k}]$ code. Then

$$
\rho(C) \leqq T[\tilde{n}, \tilde{k}] .
$$

Proof. The proof is by induction on $k$, the result being immediate for $k=0$ and 1 . Let $C$ have multiplicities $m_{P}$, and choose $Q$ so that $m_{Q}$ is odd (if all $m_{P}$ are even, $\rho(C)=0)$. Then $C_{0}^{[Q]}$ has dimension $k-1$. Let $\hat{C}$, the contracted code of $C_{0}^{[Q]}$, have parameters $[\nu, \kappa]$. Without loss of generality we may assume the generator matrix for $C$ has the form shown in Fig. 1. The parities of the multiplicities $m_{Q}, m_{R}, m_{S}, \cdots$ are indicated below the matrix. $Q R S$ is a typical line with $m_{R}, m_{S}$ odd; $Q T U$ is a typical line with $m_{T}$ odd and $m_{U}$ even; and $Q V W$ is a typical line with $m_{V}, m_{W}$ even. There are $\eta$ such pairs $R, S$, and $\nu$ such pairs $T, U . x, y, z$ denote column vectors of length $k-1$.

Then $\tilde{k}$ is the rank of the subspace of $\mathbf{F}^{k}$ spanned by the columns of types $Q, R, S$ and $T$, while $\kappa$ is the rank of the subspace of $\mathbf{F}^{k-1}$ spanned by the vectors $y$. The difference in rank $\Delta=\tilde{k}-\kappa$ therefore satisfies $1 \leqq \Delta \leqq 1+\eta$. By the pivoting bound, $\rho(C) \leqq \eta+$ $\rho\left(C_{0}^{[Q]}\right)$, and by the induction hypothesis $\rho(C) \leqq \eta+T[\nu, \kappa]$. The desired result will

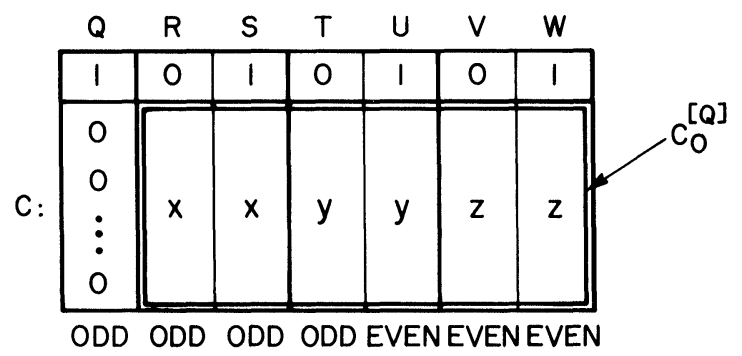

FIG. 1. Pivoting (see proof of Theorem 6). 
follow if we show that

$$
\eta+T[\nu, \kappa] \leqq T[\tilde{n}, \tilde{k}] .
$$

We also know (by counting the odd columns in Fig. 1) that $\tilde{n}=1+2 \eta+\nu$. By (23), (24) we can find a code $A$ with parameters $[2 \eta+1, \Delta] R=\eta$ (using (23) if $1 \leqq \Delta \leqq \eta$, and (24) if $\Delta=\eta+1)$. Suppose $B$ is a code with parameters $[\nu, \kappa]$ and covering radius $R=T[\nu, \kappa]$. Then $A \oplus B$ has length $1+2 \eta+\nu=\tilde{n}$, dimension $\kappa+\Delta=\tilde{k}$, and covering radius $\eta+T[\nu, \kappa]$. This implies (32) and completes the proof.

By combining Theorem 8 of [10] with Theorem 6 , we obtain our final bound. $[\tilde{n}, \tilde{k}]$. Then

THEOREM 7. Given a code $C$, let the contracted code $\tilde{C}$ have parameters

(a) $\rho(C) \leqq[\tilde{n} / 2]-1$ if $\tilde{k} \leqq[\tilde{n} / 2]$ and $\tilde{C}$ is not a simplex code;

(b) $\rho(C)=[\tilde{n} / 2] \quad$ if $\tilde{k} \leqq[\tilde{n} / 2]$ and $\tilde{C}$ is a simplex code (this requires $\tilde{n}=$ $2^{i}-1$ for some $\left.i\right)$;

(c) $\rho(C) \leqq \tilde{n}-\tilde{k} \quad$ if $\tilde{k} \geqq\lceil\tilde{n} / 2\rceil$.

Remark. We conjecture, but have not been able to prove, that Theorem 6 can be replaced by $\rho(C) \leqq T^{*}[\tilde{n}, \tilde{k}]$, where $T^{*}[\tilde{n}, \tilde{k}]$ is the largest covering radius of any projective $[\tilde{n}, \tilde{k}]$ code.

5. Behavior of $\rho$ as $\boldsymbol{n} \rightarrow \infty$; structure of best covering codes. In this section we investigate the behavior of $\rho$ as the length $n \rightarrow \infty$ (while the dimension $k$ is held fixed). There are only a finite number of projective codes of a given dimension $k$ (for their length cannot exceed $2^{k}-1$ ). Previously we began with an arbitrary code $C$ and considered the contracted code $\tilde{C}$. Now we reverse the process and begin with a projective code $B$, with parameters $\left[n_{B}, k\right]$, and consider an arbitrary $[n, k] R$ code $C$, with multiplicities $m_{i}$ say, for which $\tilde{C}=B$.

Note that $R$ and $\rho(C)$ are related via (2):

$$
\rho(C)=R-\frac{n}{2}+\frac{n_{B}}{2} .
$$

Therefore, if $n_{B}$ and $n$ are fixed, by minimizing $\rho(C)$ we minimize $R$.

If $\tilde{C}=B$ and $B$ is stable ( $(3)$, then $\rho(C)=\rho(B)$; in general, however, $\rho(C)>\rho(B)$. We define ${ }^{3}$

$$
\rho_{\infty}(B)=\max \{\rho(C): C \equiv B, C>B\} .
$$

Note that in view of Theorem 1(b), adjoining pairs of identical columns from outside the subspace of $P G(2, k-1)$ has no effect on $\rho$, and so there is no loss of generality in (34) in assuming that $\operatorname{dim} C=\operatorname{dim} B$. We also note that by $(22), \rho_{\infty}(B)$ is finite:

$$
\rho_{\infty}(B) \leqq\left[\frac{n_{B}}{2}\right] .
$$

From the pivoting bound (26),

$$
\rho_{\infty}(B) \leqq \eta+\rho_{\infty}(\hat{B}) .
$$

\footnotetext{
${ }^{3}$ This definition of $\rho_{\infty}$ differs from that in [10] in allowing even multiples of columns not present in $B$ to be adjoined to $B$.
} 
This gives a useful criterion for stability. If, for some choice of the pivot,

$$
\rho(B)=\eta+\rho_{\infty}(\hat{B}),
$$

then $B$ is stable. For (17) and (37) assume that $\rho_{\infty}(B)=\rho(B)$. Also, if $\tilde{C}=B$ and (37) holds, $C$ is tame and stable. problem:

The problem of finding $\rho_{\infty}(B)$ can also be expressed as an integer programming

$$
\rho_{\infty}(B)=\max \left\{h_{1}+\cdots+h_{2^{k}-1}\right\},
$$

subject to the constraints

$$
\begin{aligned}
& h_{i} \in \mathbf{Z} \text { for } i=1, \cdots, 2^{k}-1, \\
& \sum_{i=1}^{2^{k}-1} h_{i} c_{i} \leqq \frac{1}{2} \sum_{i=1}^{2^{k}-1} \pi_{i} c_{i} \text { for } c=\left(c_{1}, \cdots, c_{2^{k}-1}\right) \in S_{k},
\end{aligned}
$$

where the $c_{i}(=0$ or 1$)$ in (40) are interpreted as real numbers, and $\pi_{i}=0$ if $m_{i}$ is even, $\pi_{i}=1$ if $m_{i}$ is odd (cf. [10, Thm. 1]).

If $C$ is such that $\tilde{C}=B, \operatorname{dim} C=\operatorname{dim} B$, and $\rho(C)=\rho_{\infty}(B)$, we say that $C$ has been obtained by saturating $B$. Obviously a saturated code is stable.

It is sometimes useful to know how long it would take to saturate $B$, if pairs of identical columns were added to $B$ so as to drive $\rho$ up to $\rho_{\infty}(B)$ as quickly as possible. The next theorem gives an upper bound on the answer, and also on the values of the $m_{i}$ and $h_{i}$ that are required.

THEOREM 8. Let $B$ be a projective code of dimension $k$. (a) There is an $[n, k]$ code $C$ with multiplicities $m_{i} \leqq 2^{k}$ and length

$$
n \leqq 2^{k}\left(2^{k}-1\right),
$$

satisfying $\tilde{C}=B$ and $\rho(C)=\rho_{\infty}(B)$. (b) Consider any $[n, k]$ code $C$ with $\tilde{C}=B$ and $\rho(C)=\rho_{\infty}(B)$. Let $x$ be a deep hole in $C$ for which 0 is a closest codeword. Then the heights $h_{i}$ of the blocks of $x$ satisfy

$$
\left|h_{i}\right| \leqq 2^{k-1}, \quad i=1, \cdots, 2^{k}-1 .
$$

Proof. We prove (b) first. We know that

$$
h_{1}+\cdots+h_{2^{k}-1}=\rho_{\infty}(B),
$$

so we shall maximize $h_{i}$ (and later $-h_{i}$ ) subject to the constraints (39), (40), (43). Inequality (40) implies that

$$
\sum_{i=1}^{2^{k}-1} h_{i} c_{i} \leqq 2^{k-2} \quad \text { for } c \in S_{k}
$$

since all the nonzero codewords in $S_{k}$ have weight $2^{k-1}$, and $\pi_{i} \leqq 1$. If the inequalities (44) corresponding to those codewords $c=\left(c_{1}, \cdots, c_{2^{k}-1}\right) \in S_{k}$ with $c_{1}=1$ are summed we obtain $2^{k-1} h_{1}+2^{k-2}\left(h_{2}+\cdots+h_{2^{k}-1}\right) \leqq 2^{2 k-3}$, which by (43) becomes $h_{1} \leqq 2^{k-1}-\rho_{\infty}(B) \leqq 2^{k-1}$. Similarly, by using the codewords with $c_{1}=0$, we obtain $-h_{1} \leqq 2^{k-1}$, so $\left|h_{1}\right| \leqq 2^{k-1}$. The same argument applies to the other $h_{i}$. (a) Knowing the possible range of the individual $h_{i}$ we can work out how large the multiplicities $m_{i}$ must be. From (9), $m_{i}=2^{k}$ is enough to permit $-2^{k-1} \leqq h_{i} \leqq 2^{k-1}$. Therefore there is a code $C$ of length $n=\sum m_{i} \leqq 2^{k}\left(2^{k}-1\right)$ with $\rho(C)=\rho_{\infty}(B)$. 
Theorem 8 implies that we can add the extra inequalities

$$
\left|h_{i}\right| \leqq 2^{k-1}, \quad i=1, \cdots, 2^{k}-1,
$$

to the integer program (38)-(40) defining $\rho_{\infty}(B)$. Stronger bounds than (45) can often be obtained in particular cases by detailed examination of $(40)$.

The quantity $\rho_{\infty}(B)$ tells us the maximal value of $\rho(C)$ over all codes $C$ with $\tilde{C}=B$. We now investigate the minimal value. Let

$$
\rho_{n}^{*}(B)=\min \{\rho(C): C \text { is an }[n, k] \text { code with } C \equiv B, C \geqq B\} .
$$

In view of (33), this also minimizes the covering radius of $C$ over all $[n, k]$ codes with $\tilde{C}=B$. From $\rho_{n}^{*}(B) \leqq \rho_{\infty}(B)$ and $(15)$, for sufficiently large $n \rho_{n}^{*}(B)$ is independent of $n$, and will be denoted by $\rho_{*}(B)$. Then

$$
\rho(B) \leqq \rho_{*}(B) \leqq \rho_{\infty}(B) .
$$

THEOREM 9. Let $B$ be an $\left[n_{B}, k\right]$ projective code. For sufficiently large $n$ of the same parity as $n_{B}$ there is an $[n, k]$ normal code $C$ with $C \equiv B, C \geqq B$, and $\rho(C)=\rho_{*}(B)$, which is obtained by adjoining $n-n_{B}$ copies of a single column to $B$.

Proof. Let us arrange the coordinates of $S_{k}$ so that the coordinates of $B$ appear first. The set of vectors $\left(h_{1}, \cdots, h_{2^{k}-1}\right)$ satisfying (39), (40) (with $\pi_{i}=1$ for $1 \leqq i \leqq n_{B}$, $\pi_{i}=0$ otherwise) and (45) is a finite set $H$ (depending on $B$ but not on $n$ ).

For fixed large $n$ with $n \equiv n_{B}(\bmod 2)$, we consider all $[n, k]$ codes $C$ for which $\tilde{C}=B$. Such a code $C$ is defined by its multiplicities $m_{1}, \cdots, m_{2^{k}-1}$, where

$$
\begin{aligned}
& m_{1}, \cdots, m_{n_{B}} \text { are odd, } \\
& m_{n_{B}+1}, \cdots, m_{2^{k}-1} \text { are even, and } \\
& \sum_{i=1}^{2^{k}-1} m_{i}=n .
\end{aligned}
$$

Then $\rho(C)=\rho^{(k)}\left(m_{1}, \cdots, m_{2^{k}-1}\right)$ is equal to the maximum of $h_{1}+\cdots+h_{2^{k}-1}$ subject to (39), (40) and

$$
-\left[\frac{m_{i}}{2}\right] \leqq h_{i} \leqq\left\lceil\frac{m_{i}}{2}\right\rceil \text { for } i=1, \cdots, 2^{k}-1
$$

(cf. [10, Thm. 1]). Also

Let us define

$$
\rho_{n}^{*}(B)=\min \left\{\rho^{(k)}\left(m_{1}, \cdots, m_{2^{k}-1}\right): \text { the } m_{i} \text { satisfy }(48)\right\} .
$$

$$
f(i)=\rho^{(k)}\left(1, \cdots, 1, n-n_{B}+1,1, \cdots, 1,0, \cdots, 0\right),
$$

for $i=1, \cdots, n_{B}$, where $n-n_{B}+1$ is in position $i$ and there are $n_{B}-1$ ones, and

$$
f(i)=\rho^{(k)}\left(1, \cdots, 1,0, \cdots, 0, n-n_{B}, 0, \cdots, 0\right)
$$

for $i=n_{B}+1, \cdots, 2^{k}-1$, where $n-n_{B}$ is in position $i$ and there are $n_{B}$ ones. Since $H$ is a finite set, for $n$ sufficiently large $f(i)$ is independent of $n$ and is equal to either

$$
\rho^{(k)}\left(1, \cdots, 1, a_{i}, 1, \cdots, 1,0, \cdots, 0\right)
$$

for $i=1, \cdots, n_{B}$, or

$$
\rho^{(k)}\left(1, \cdots, 1,0, \cdots, 0, a_{i}, 0, \cdots, 0\right)
$$

for $i=n_{B}+1, \cdots, 2^{k}-1$, where $a_{1}, \cdots, a_{2^{k}-1}$ are constants (depending only on $B$ ). 
Finally, let

$$
M=\min \left\{f(1), \cdots, f\left(2^{k}-1\right)\right\}
$$

and choose an $r$ such that $f(r)=M$.

After these preliminaries we come to the heart of the proof. Suppose $n$ is large (specifically, we need $\left.n>2^{k} \max \left\{a_{i}\right\}\right)$, and $\rho_{n}^{*}(B)=\rho^{(k)}\left(m_{1}, \cdots, m_{2^{k}-1}\right)$, where the $m_{i}$ satisfy (48). At least one of the $m_{i}$ must be large, say $m_{j}$ (and in particular $m_{j}>a_{j}$ ). By the monotonicity property (15),

$$
\begin{aligned}
\rho_{n}^{*}(B) & \geqq \begin{cases}\rho^{(k)}\left(1, \cdots, m_{j}, \cdots, 1,0, \cdots, 0\right) & \left(j \leqq n_{B}\right) \\
\rho^{(k)}\left(1, \cdots, 1,0, \cdots, m_{j}, \cdots, 0\right) & \left(j>n_{B}\right)\end{cases} \\
& = \begin{cases}\rho^{(k)}\left(1, \cdots, a_{j}, \cdots, 1,0, \cdots, 0\right) & \left(j \leqq n_{B}\right) \\
\rho^{(k)}\left(1, \cdots, 1,0, \cdots, a_{j}, \cdots, 0\right) & \left(j>n_{B}\right)\end{cases} \\
& \geqq M .
\end{aligned}
$$

In fact $\rho_{n}^{*}(B)=M$, since if $C$ has multiplicities $m_{i}=1$ for $i \leqq n_{B}$ and $m_{i}=0$ for $i>n_{B}$, except for $m_{r}=n-n_{B}+1$ (if $r \leqq n_{B}$ ) or $n-n_{B}$ (if $r>n_{B}$ ), then $\rho(C)=M$. Therefore $\rho_{n}^{*}(B)=\rho_{*}(B)=M$.

Since $\rho(C)=\rho_{*}(B)$, we can adjoin $2 l$ copies of the special column to $C$ without increasing $\rho$, for $l=0,1, \cdots$. Therefore $C$ is normal by Lemma 5 . This completes the proof of Theorem 9.

Remark. The single column mentioned in the theorem need not be a column of $B$.

Example. As an illustration of the preceding notions we analyze the $[11,5] R=3$ code $C_{11}^{\prime}$ having generator matrix

$C_{11}^{\prime}:$\begin{tabular}{lllllllllll|}
1 & 2 & 3 & 4 & 5 & 6 & 7 & 8 & 9 & 10 & 11 \\
\hline 1 & 0 & 0 & 0 & 0 & 0 & 1 & 1 & 1 & 1 & 1 \\
0 & 1 & 0 & 0 & 0 & 1 & 1 & 0 & 0 & 0 & 1 \\
0 & 0 & 1 & 0 & 0 & 1 & 0 & 1 & 0 & 0 & 1 \\
0 & 0 & 0 & 1 & 0 & 1 & 0 & 0 & 1 & 0 & 1 \\
0 & 0 & 0 & 0 & 1 & 1 & 0 & 0 & 0 & 1 & 1
\end{tabular},

which is an interesting code for several reasons (see $\S 6$ ). It has weight distribution $0^{1} 4^{10} 6^{16} 8^{5}$, and its automorphism group has order 1920 , with two orbits on coordinates, $\{1\}$ and $\{2, \cdots, 11\}$. Suppose $C$ is an $[n, 5]$ code with $\tilde{C}=C_{11}^{\prime}$, having odd multiplicities $m_{1}, \cdots, m_{11}$ on the columns of (50), and even multiplicities on the remaining 20 nonzero columns of length 5 . From $(17), \rho(C) \geqq \rho\left(C_{11}^{\prime}\right)=3$.

If we pivot on coordinate $Q=1$, we see that $\eta=5$ (since columns 2 and 7,3 and 8 , etc. combine), $C_{0}^{[1]}$ has even multiplicities; i.e., $\hat{C}=0$, and (26) states that $\rho(C) \leqq 5$. On the other hand, if we pivot at $Q=2$ we find that $\eta=1, \hat{C}$ is the $[8,4]$ extended Hamming code, for which $\rho=2$ or 3 depending on the multiplicities $m_{P}^{\prime}$ [10, Thm. 16], and (26) yields $\rho(C) \leqq 4$. We conclude that

$$
3 \leqq \rho(C) \leqq 4 .
$$

The following theorem determines which of these two possibilities occurs.

THEOREM 10. Let $C$ be any code for which the contracted code is the $[11,5] R=3$ code $C^{\prime}{ }_{11}$ defined by (50). If column 1 of $(50)$ has multiplicity $\geqq 3$ or any of the 20 nonzero 
columns of length 5 not in (50) have positive multiplicity, then $\rho(C)=4$; otherwise $\rho(C)=3$. Thus $\rho_{*}\left(C_{11}^{\prime}\right)=3, \rho_{\infty}\left(C_{11}^{\prime}\right)=4$.

Proof. To check the first assertion we verify (by computer) that if two copies of column 1 or of any of the 20 missing columns are adjoined to (50), $\rho$ increases to 4 , and then we use monotonicity (15). On the other hand, by integer programming we find that $\rho(C)=3$ in all other cases.

For example, by assigning multiplicities $1,1,1,1, m, 1, \cdots, 1$ ( $m$ odd) to the columns of (50) we obtain an infinite sequence of $[n=m+10,5]$ codes with $\rho=3$ and covering radius $R=3+[m / 2]=(n-5) / 2$, for odd $n \geqq 11$. Figure 2 shows the case of length 23. These codes are optimal coverings, for it is proved in Theorem 22 of [3] that $t[n, 5]=[(n-5) / 2](n \neq 6)$. (They are not unique, however; there are many codes that achieve this bound).

Remark. Definitions (34), (46) and Theorem 9 still apply if $B$ is not projective (although the proof of Theorem 9 must be modified slightly).

THEOREM 11. If $C$ is normal, then $\rho_{*}(C)=\rho(C)$.

Proof. Suppose column $i$ of $C$ is acceptable. By adjoining $2 l$ copies of column $i$ to $C$ we obtain a code $D$ with $\rho(D)=\rho(C)$ (see Theorem 2). Therefore $\rho_{*}(C)=\rho(C)$.

Remark. Similarly, if $C$ is a code of length $n$ with the property that, for all $i=1, \cdots, n$, adjoining two copies of column $i$ to $C$ increases $\rho$, then $C$ is abnormal.

Theorem 9 also provides information about the best possible covering codes.

THEOREM 12. For fixed $k$, and all sufficiently large $n$, then (a)

$$
t[n, k]=\frac{n}{2}+\min _{B}\left\{\rho_{*}(B)-\frac{n_{B}}{2}\right\},
$$

where $B$ ranges over all projective codes of dimension $k$ or $k-1$ (a finite set), and $n_{B}$ is the length of $B$; (b) there is a normal $[n, k] R$ code $C$ with $R=t[n, k]$ in which all columns have multiplicity 1 except for one column which has large multiplicity; and (c)

$$
t[n+2, k]=t[n, k]+1 \text {. }
$$

Proof. Suppose $n \gg k$, and let $\mathscr{C}[n, k]$ denote the set of all $[n, k]$ codes with covering radius $R=t[n, k]$. Choose any $C \in \mathscr{C}[n, k]$ and let $B=\tilde{C}$ be an $\left[n_{B}, k_{B}\right]$ code. Then $\rho(C) \geqq \rho_{*}(B)$. By Theorem 9 there is an $\left[n-2\left(k-k_{B}\right), k_{B}\right]$ normal code $D$ with $\tilde{D}=B$ and $\rho(D)=\rho_{*}(B)$. Then $C^{\prime}=D \oplus T_{2}^{k-k_{B}}$ is an $[n, k]$ normal code with contracted code $B$, and $\rho\left(C^{\prime}\right)=\rho_{*}(B)$. Thus $C^{\prime} \in \mathscr{C}[n, k]$ and $\rho(C)=\rho\left(C^{\prime}\right)=$ $\rho_{*}(B)$. From (2), $C R(C)=\frac{1}{2} n-\frac{1}{2} n_{B}+\rho_{*}(B)$, and therefore

$$
t[n, k]=\frac{n}{2}+\min _{B}\left\{\rho_{*}(B)-\frac{n_{B}}{2}\right\},
$$

where $B$ ranges over all projective codes of dimension $k_{B} \leqq k$.

We next show that in fact $k_{B}=k$ or $k-1$, and that there is a normal code $C^{\prime \prime} \in \mathscr{C}[n, k]$ in which all but one of the columns has multiplicity 1 .

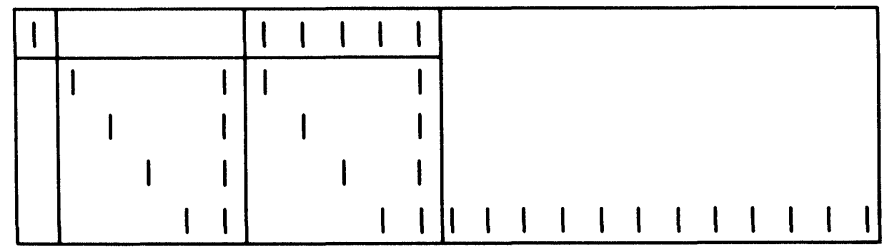

FIG. $2 . A[23,5] R=9$ optimal covering code obtained from (50). Blank entries indicate zeros. 
Case $(\mathrm{i}): k \equiv k_{B}(\bmod 2)$. By Theorem 9 there is an $\left[n-\left(k-k_{B}\right), k_{B}\right]$ normal code $A$ with $\tilde{A}=B$ and $\rho(A)=\rho_{*}(B)$, obtained by adjoining $\left(n-n_{B}\right)-\left(k-k_{B}\right)$ copies (an even number) of a single column $\beta$ (say) to $B$. Then $C^{\prime \prime}=A \oplus \mathbf{F}^{k-k_{B}}$ is an $[n, k]$ normal code with $\rho\left(C^{\prime \prime}\right)=\rho_{*}(B)$, and has $n_{B}+k-k_{B}$ distinct columns with odd multiplicity. From (2),

$$
C R\left(C^{\prime \prime}\right)=\frac{n}{2}-\frac{n_{B}+k-k_{B}}{2}+\rho_{*}(B)
$$

which is less than $C R(C)$ unless $k=k_{B}$. Therefore $k=k_{B}$, and $C^{\prime \prime} \in \mathscr{C}[n, k]$ has the desired multiplicities.

Case (ii): $k \not \equiv k_{B}(\bmod 2)$. By Theorem 9, for sufficiently large $N_{0}$ there is an $\left[N_{0}, k_{B}\right]$ normal code $A_{0}$ with $\tilde{A}_{0}=B$ and $\rho\left(A_{0}\right)=\rho_{*}(B)$, obtained by adjoining $N_{0}-n_{B}$ copies of a single column $\beta$ to $B$. Let $A_{i}$ be obtained by adjoining $i$ further copies of $\beta$ to $A_{0}$. We know $C R\left(A_{2}\right)=C R\left(A_{0}\right)+1$, so

$$
\begin{array}{lll}
\text { either } & C R\left(A_{1}\right)=C R\left(A_{0}\right), & C R\left(A_{2}\right)=C R\left(A_{1}\right)+1, \\
\text { or } & C R\left(A_{1}\right)=C R\left(A_{0}\right)+1, & C R\left(A_{2}\right)=C R\left(A_{1}\right) .
\end{array}
$$

In the first case we call $A_{0}$ late and in the second case we call it early. Whether $A_{0}$ is late or early depends on the solution to a certain integer programming problem. Therefore, in the sequence $A_{0}, A_{2}, A_{4}, \cdots$, from a certain point on either all $A_{2 i}$ are early or all are late, and similarly in the sequence $A_{1}, A_{3}, A_{5}, \cdots$. Thus for sufficiently large $i, A_{2 i+1}$ satisfies the hypothesis of Lemma 5 and is normal. In particular, by taking

$$
i=\frac{1}{2}\left\{n-1-N_{0}-\left(k-k_{B}\right)\right\}
$$

we obtain normal codes $A_{2 i}$ and $A_{2 i+1}$ of dimension $k_{B}$ and lengths $n-1-\left(k-k_{B}\right)$ and $n-\left(k-k_{B}\right)$, respectively, with $\rho\left(A_{2 i}\right)=\rho_{*}(B)$,

$$
\begin{aligned}
& C R\left(A_{2 i}\right)=\frac{n-1-\left(k-k_{B}\right)}{2}-\frac{n_{B}}{2}+\rho_{*}(B), \\
& C R\left(A_{2 i+1}\right) \leqq C R\left(A_{2 i}\right)+1 .
\end{aligned}
$$

Finally, let $C^{\prime \prime}=A_{2 i+1} \oplus \mathbf{F}^{k-k_{B}}$. Then

$$
C R\left(C^{\prime \prime}\right)=C R\left(A_{2 i+1}\right) \leqq \frac{n}{2}-\frac{n_{B}}{2}+\rho_{*}(B),
$$

since $k \geqq k_{B}+1$, with equality only if $k=k_{B}+1$ and $A_{2 i}$ is early. Therefore $k=k_{B}+$ 1 , and $C^{\prime \prime} \in \mathscr{C}[n, k]$ has the desired multiplicities. This completes the proof of (a) and (b). To prove (c) we observe that the best choice for $B$ in (52) is independent of $n$, and when $n$ increases to $n+2$, the right-hand side of (52) increases by 1 .

Remarks. (1) This theorem establishes Conjectures A and D of [2] for sufficiently large $n$. (2) If the optimal code $C$ is obtained by adjoining $2 l$ columns to $B$, then $C$ has length $n=n_{B}+2 l$ and covering radius $R=\rho_{*}(B)+l$. We can write this as

$$
\frac{n}{2}-R=\frac{n_{B}}{2}-\rho_{*}(B)
$$

or, if $B$ is normal, as

$$
\frac{n}{2}-C R(C)=\frac{n_{B}}{2}-C R(B)
$$


Thus the best seed codes $B$ are normal codes for which the parameter

$$
\delta=\frac{n_{B}}{2}-C R(B)
$$

is as large as possible. (3) A possible interpretation of Theorems 9 and 12 is the following. An optimal covering code has the property that the codewords are constructed so as to be not too far from an arbitrary $n$-tuple $x$. This is a difficult task for $n \gg k$, since we are using only $2^{k}$ vectors to cover $2^{n}$ vectors. We may say informally that codes with the structure described in Theorem 12(b) do this by matching $x$ very carefully on a small number $\left(n_{B}\right)$ of coordinates, and just using an average on the rest (see Fig. 2). (4) This special structure also greatly simplifies the process of finding the closest codeword to a given $x$.

\section{REFERENCES}

[1] G. D. Cohen, M. G. Karpovsky, H. F. MAtTson, JR. AND J. R. SChAtz, Covering radius-survey and recent results, IEEE Trans. Inform. Theory, IT-31 (1985), pp. 328-343.

[2] G. D. Cohen, A. C. LoBSTEIN AND N. J. A. SLOANE, Further results on the covering radius of codes, IEEE Trans. Inform. Theory, IT-32 (1986), pp. 680-694.

[3] R. L. Graham AND N. J. A. Sloane, On the covering radius of codes, IEEE Trans. Inform. Theory, IT-31 (1985), pp. 385-401.

[4] H. J. Helgert AND R. D. STINAFF, Minimum-distance bounds for binary linear codes, IEEE Trans. Inform. Theory, IT-19 (1973), pp. 344-356.

[5] J. S. LEON, Computing automorphism groups of error correcting codes, IEEE Trans. Inform. Theory, IT-28 (1982), pp. 496-511.

[6] F. J. MacWilliams and N. J. A. Sloane, The Theory of Error-Correcting Codes, North-Holland, Amsterdam, 1977.

[7] H. F. MATTSON, JR., An Improved Upper Bound on Covering Radius, Lecture Notes Computer Science 228, 1986, pp. 90-106.

[8] D. SlePIAN, Some further theory of group codes, Bell System Tech. J., 39 (1960), pp. 1219-1252.

[9] N. J. A. SLOANE, Unsolved problems related to the covering radius of codes, in Proc. Specific Problems in Communication and Computation (SPOC-85), Springer-Verlag, New York, 1987, to appear.

[10] — A new approach to the covering radius of codes, J. Combin. Theory Ser. A, 42 (1986), pp. 61-86.

[11] T. VERHOEFF, Updating a table of bounds on the minimum distance of binary linear codes, Math. Dept., Eindhoven Univ. Tech., Report 85-WSK-01, Eindhoven, the Netherlands, January 1985; IEEE Trans. Inform. Theory, to appear. 